\title{
Agreement of Central Corneal Thickness Measurements Using two Different Ultra Sound Techniques
}

\author{
Dr Karthika Mohankumar ${ }^{1}$ Dr. Rashmi Jain ${ }^{2}$, Dr Vidya Hegde, \\ Dr Anupama B, Dr. Rashmi S \\ ${ }^{I}$ (Ophthalmology, Yenepoya Medical College, India) \\ ${ }_{2}^{2}$ (Ophthalmology, Yenepoya Medical College, India)
}

\begin{abstract}
Purpose: To compare the Central Corneal Thickness (CCT) measurements obtained by Ultrasound Pachymeter (USP) \& Ultrasound Biomicroscope (UBM).

Methods: The study CCT measurements of 60 eyes was measured using first with UBM followed by USP. Results were compared statistically using Bland-Altman plots \& Deming's regression analysis

Results: Scatter plot analysis showed that for the same value of CCT UBM, corresponding CCTP measurements vary systematically. Bland - Altman plot analysis showed that there is systematic difference between the measurements with both instruments. Deming's regression analysis confirmed a reasonable agreement between both two measurements.

Conclusion: There is a positive correlation in our study \& the readings obtained by UBM correlate with that measured by Pachymeter. But since the interobserver variability is high with UBM, pachymeter is considered to be a better modality of measurement of CCT among the two of them.
\end{abstract}

Keywords: Central corneal thickness, Ultrasound Pachymeter,Ultrasound Biomicroscope

\section{Introduction}

Accurate measurement of central corneal thickness (CCT) is of clinical, diagnostic and surgical importance in Ophthalmic practice. The measurement of CCT has varied applications in intraocular pressure (IOP) estimation, screening for corneal surgeries, evaluation of cases contact lens related complications and in corneal decompensation. It even has a prognostic role in cases of corneal degenerations \& post LASIK ectasia. The normal corneal thickness ranges from $0.49-0.56 \mathrm{~mm}$ at the centre and $0.7-0.9 \mathrm{~mm}$ at the limbus. The mean CCT as shown by various studies is $0.51-0.52 \mathrm{~mm}$ (standard deviation $0.02-0.04 \mathrm{~mm}$ ). ${ }^{[1]}$ The cornea is significantly thicker in the age group of 40-80 years than in individuals below 40 years. It has also been found to be higher in males \& diabetic patients. ${ }^{[2]}$ CCT has no correlation with refraction or systemic hypertension. Peripheral corneal thickness is asymmetric with the temporal cornea being the thinnest followed by the inferior cornea. The CCT reading of $0.7 \mathrm{~mm}$ or more is indicative of endothelial decompensation. ${ }^{[3]}$

There are several ways of assessing CCT. This study was conducted to compare the CCT by means of two different modalities which uses ultrasound energy for measurement - Pachymeter and Biomicroscope.

\section{Materials And Methods}

Institutional Ethics committee approval was obtained for the study. A hospital based, cross sectional, comparative study was conducted at a tertiary medical college hospital. Participants were enrolled from patients attending the Ophthalmology outpatient department. The patients who had congenital ocular anomalies, history of corneal diseases (ulcer/ epithelial defect/ opacity), contact lens users, on any topical medications, history of ocular trauma, underwent ocular surgery or any LASER treatment, were excluded from the study. Eligible participants of either gender, above the age of 18 years were explained the need of study, the procedure of examination, tests involved and a written informed consent obtained. The consenting eligible participants were enrolled for the study. Detailed history \& comprehensive ocular examination was performed. CCT was measured with both an Ultrasound Pachymeter \& Ultrasound Biomicroscope by the same observer.CCT was measured first with UBM (MARVEL- GBK2YC1). UBM will be done with the patient in the supine position and the eye open. Patient is made to comfortably lie down in a couch. After entering the baseline patient data in the UBM machine, patient's eye to be examined will be anaesthetised with Proparacaine $0.5 \%$ eye drops. A cup of appropriate size which fits in between the eyelids is used. It will be filled with either $0.9 \%$ saline or sterile methylcellulose. The probe of the transducer is placed in saline approximately $2 \mathrm{~mm}$ from the eye surface. This distance prevents injury to the cornea and also helps as a fluid standoff. The image is captured \& CCT measured using linear scale tool. Followed by which, CCT was measured with Ultrasound Pachymetry (Sonomed 200PT). Cornea was anaesthetized with $0.5 \%$ Proparacaine hydrochloride ophthalmic solution. Pachymeter probe was 
placed perpendicularly on the central cornea. This was confirmed by an audible beep produced by the instrument. Average of five readings was taken as the final value of measurement.

Ultrasound Pachymetry (USP) operates at frequencies of 20 to $50 \mathrm{MHz}$ and emits short acoustic pulses and detects reflections from the anterior and posterior surfaces of the cornea. Corneal thickness is calculated from the measured time-of-flight between these reflections and the accepted speed of sound in the cornea of $1636-1640 \mathrm{~m} / \mathrm{s}^{[4]}$

Ultrasound Biomicroscope (UBM) uses a high frequency (35-50 MHz) ultrasound beam, with a depth of penetration of $5 \mathrm{~mm} \&$ image resolution of $50 \mu \mathrm{m} .{ }^{[5]}$ The images were captured and measurements made using linear scale tool. The image should be well centralized for a clear resolution.

\section{Results}

A total of 60 eyes were examined. This included 35 males and 25 females. Age ranged from 20-

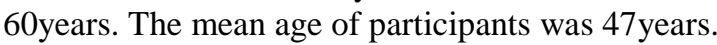

\section{Figure 1 depicts a Scatter Plot depicting correlation of central corneal thickness with pachymeter \& UBM}

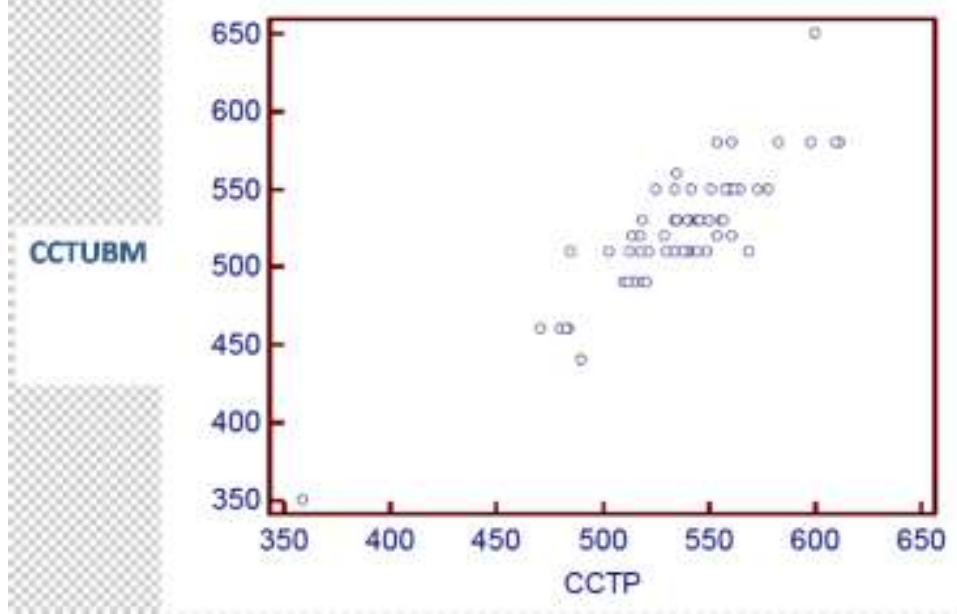

Figure 1

The dotted line in the above graph is the line of agreement. For the same value of CCT UBM , corresponding CCTP measurements vary systematically.

Figure 2 showing Bland-Altman-plot depicting mean CCTP \& CCTUBM

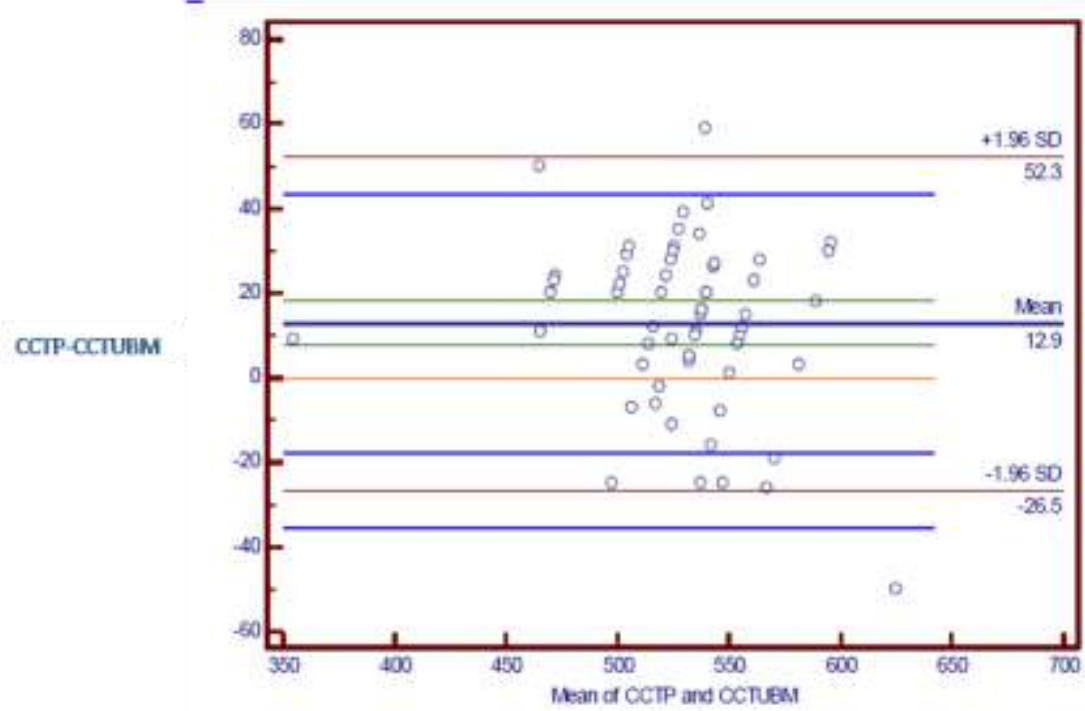

Figure 2

The analysis was carried out using MedCalc ver.15.6.1. X Axis shows Mean of CCT of measurements with ultrasound Pachymeter \& UBM. Y Axis - Difference of measurements with Ultrasound Pachymeter \& UBM. In 
Bland-Altman plot the values lie between the measures of agreement. Hence we can say that there is reasonable agreement between CCTP and CCTUBM. The zero-line( line of equality) falls outside the confidence interval, which indicates systematic difference.

Figure 3 shows Deming Regression graph to show the difference between the two methods

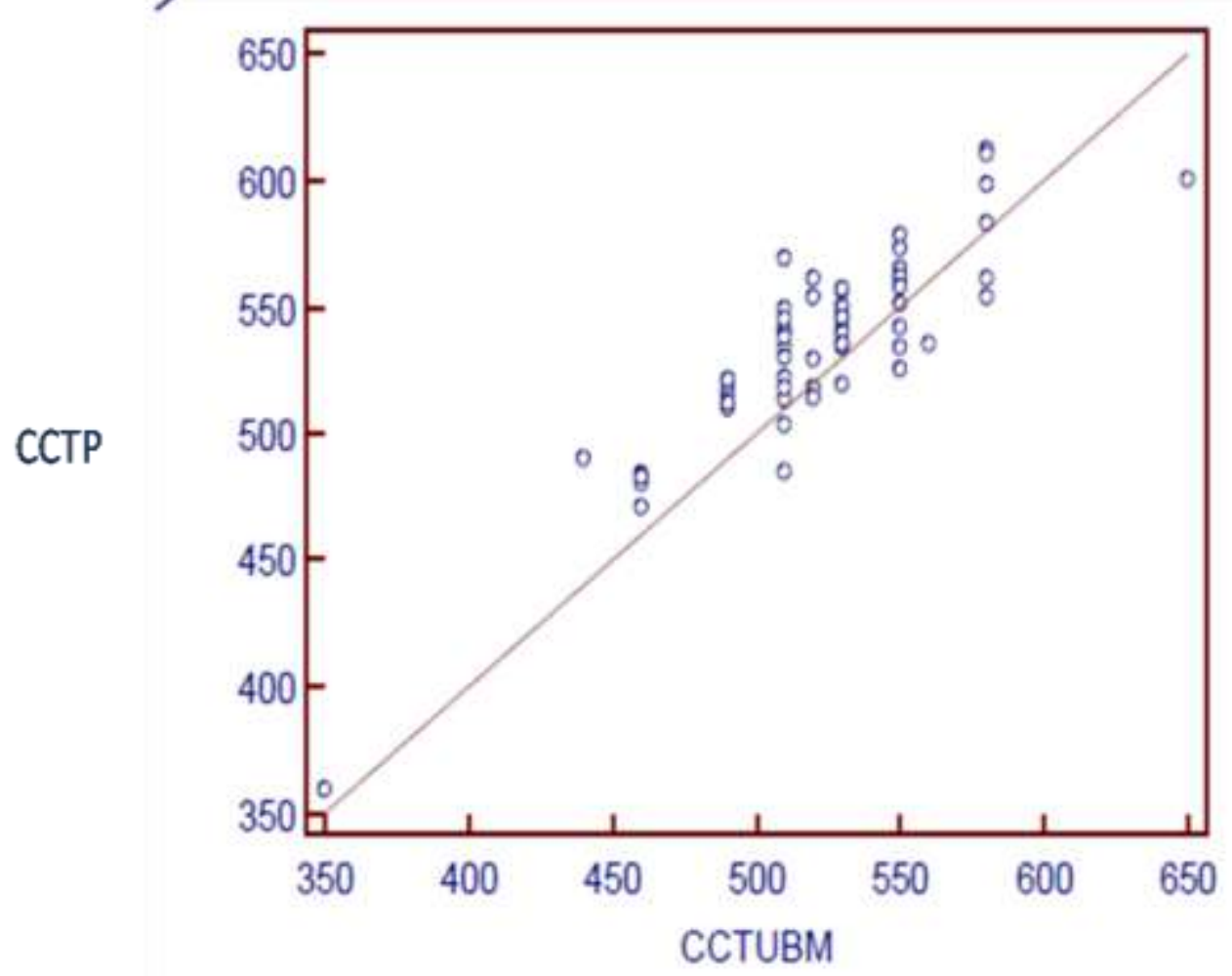

The intercept value is 68.7860 with confidence interval including 0 . This value measures that the systematic difference between the two methods is equal to 0. I.e. we accept the hypothesis that intercept is 0 . The slope coefficient is 0.8931 with confidence interval including 1 . This indicates that the proportional difference between the 2 methods is equal to $1(\mathrm{i} . \mathrm{e}, \mathrm{Y}=\mathrm{X})$. We accept the hypothesis that slope is equal to 1 . Hence from Deming regression and plots we can conclude that there is reasonable agreement between the 2 measurements. The regression equation can be validated using an independent set of observations. The equation can be used to predict the CCTP measurement corresponding to an observed CCTUBM measurement.

\section{Discussion}

The measurement of CCT plays an important role in several clinical situations, where incorrect CCT value can lead to an erroneous diagnosis or management.

In IOP assessment CCT determines the correction factor. CCT is a valuable parameter in patients with glaucoma. CCT bears an inverse relation with the risk of developing glaucomatous damage. ${ }^{[6]}$ Screening and surgical management plan of patients for keratorefractive procedures like radial keratotomy \& astigmatic keratotomy depends on CCT. Assessment of corneal thickness and edema is of significance in Contact lens wearers and Corneal ectasias.

There are various techniques of assessing corneal thickness. Ultrasound Techniques which most commonly in use are Conventional Ultrasonic Pachymetry \& Ultrasound Biomocroscopy (UBM). ${ }^{[7]}$ Optical Techniques include Manual optical pachymetry, Specular microscopy, ${ }^{[8]}$ Scanning slit lamp corneal topography, ${ }^{[9]}$ Optical Coherence Tomography, ${ }^{[10]}$ Optical low coherence interferometry, ${ }^{[11]}$ Confocal microscopy, ${ }^{[12]}$ Laser Doppler interferometry. Recent techniques like Pentacam, Pachycam, Ocular Response Analyzer (ORA) are also in use.

USP is the most common \& has been considered since many years as the gold standard machine for detection of CCT. ${ }^{[13-16]}$ This method has been reported to have a high degree of intraoperator and interoperator reproducibility. ${ }^{[4]}$ Placement of the probe on the corneal center can lead to operator-dependent errors due to offcenter placement (thicker measurements), ${ }^{[17,18]}$ indentation (thinner readings) ${ }^{[19]}$ are the drawbacks of pachymeter. Disadvantages like patient discomfort, epithelial damage and risk of infection also exist. 
The Ultrasound Biomicroscope (UBM) can be used to measure various parameters in the eye. There are studies which showed that the intra observer reproducibility was high for all measurements of CCT. ${ }^{[7,20]}$ In addition, there are reports which showed that there was no statistically significant difference between the mean CCT measured with the anterior segment optical coherence tomograph (AS-OCT) and the UBM. ${ }^{[21]}$ They also reported that the interobserver reproducibility for the measured parameters varied considerably and was affected by the subjective interpretation of visualized anatomic landmarks. ${ }^{[2]}$

In our study the correlation between CCT measurements with pachymeter \& UBM; that is two modalities of ultrasound techniques have been studied \& we found a good correlation in Blandt Altmann analysis. But since the inter \& intraobserver variability is high in UBM, Pachymeter values were taken as standard \& UBM values were compared to that.

There is a positive correlation in our study \& the readings obtained by UBM correlate with that measured by Pachymeter.

\section{Conclusion}

There is a correlation between values obtained by Ultrasound Pachymeter \& Ultrasound Biomicroscope. But due to high interobserver variability of UBM, most widely used technique is USP.

\section{References}

[1]. Ehlers N, BramsenT, Sperling S. Applanation tonometry and central corneal thickness. Acta Ophthalmol (Copenh) 1975;53:34-43.

[2]. Goldich Y, Barkana Y, Gerber Y, Rasko A, Morad Y, Harstein M, Avni I, Zadok D. Effect of diabetic mellitus on biomechanical parameters of the cornea. J Cataract Refract Surg2009;35(4):715-9.

[3]. Sachdev MP, Honavar SG, Thakar M. Diagnostic tests for corneal diseases. Ind J Ophthalmol 1994;42:88-89.

[4]. Miglior S, Albe E, Guareschi M, Mandelli G, Gomarasca S, Orzalesi N. Intraobserver and interobserver reproducibility in the evaluation of ultrasonic pachymetry measurements of central corneal thickness. Br J Ophthalmol 2004;88:174-7.

[5]. Silverman RH. High-resolution ultrasound imaging of the eye - a review. Clin Experiment Ophthalmol. 2009 January;37(1):54-67.

[6]. Whitacre MM, Stein RA, Hassanein K. The effect of corneal thickness on applanation tonometry. Am J Ophthalmol 1993;115:592-6.

[7]. Tam ES, Rootman DS. Comparison of central corneal thickness measurements by specular microscopy, ultrasound pachymetry, and ultrasound biomicroscopy. J Cataract Refract Surg 2003;29:1179-1184.

[8]. Bovelle R, Kaufman SC, Thompson HW, et al. Corneal thickness measurements with the Topcon SP-2000p specular microscope and an ultrasound pachymeter. Arch Ophthalmol 1999;117:868-870.

[9]. Yaylali V, Kaufman SC, Thompson HW. Corneal thickness measurements with the orbscan topography system and ultrasound pachymetry. J Cataract Refract Surg 1997;23:1345-1350.

[10]. Bechman M, Thiel MJ, Neubauer AS, et al. Central corneal thickness measurements with a retinal optical coherence tomography device versus standard ultrasonic pachymetry. Cornea 2001;20:50-54.

[11]. Rainer G, Findl O, Petternel V, et al. Central corneal thickness measurements with partial coherence interferometry, ultrasound, and the Orbscan System. Ophthalmology 2004;111:875-879.

[12]. Li HF, Petroll WM, Møller-Pederson T, et al. Epithelial and corneal thickness measurements by in vivo confocal microscopy through focusing (CMTF). Curr Eye Res 1997;16:214-221.

[13]. Barkana Y, Gerber Y, Elbaz U, Schwartz S, Ken-Dror G, Avni I, Zadok D. CCT measurements with the Pentacam Scheimpfl ug system, optical lowcoherence refl ectometry pachymeter and USP. J Cataract Refract Surg 2005; 31:1729-1735.

[14]. Yazici AT, Bozkurt E, Alagoz C, Pekel G, Kaya V, Yilmaz OF. CCT, anterior chamber depth, and pupil diameter measurements using Visante OCT, Orbscan and Pentacam. J Cataract Refract Surg 2010; 26:12 6-137.

[15]. Gordon A, Boggess EA, Molinari JF. Variability of USP. Optom Vis Sci 1990; 67:16 2-165.

[16]. Marisch MM, Bullimore MA. The repeatability of corneal thickness measures. Cornea 2000; 19:79 2-795

[17]. Buehl W, Stojanac D, Sacu S, Drexler W, Findl O. Comparison of three methods of measuring corneal thickness and anterior chamber depth. Am J Ophthalmol. 2006;141:7-12.

[18]. Findl O, Kriechbaum K, Sacu S, Kiss B, Polak K, Nepp J, et al. Influence of operator experience on the performance of ultrasound biometry compared to optical biometry before cataract surgery. J Cataract Refract Surg. 2003;29:1950-5

[19]. Solomon OD. Corneal indentation during ultrasonic pachymetry. Cornea. 1999;18:214-5.

[20]. Urbak SF, Pedersen JK, Thorsen TT. Ultrasound biomicroscopy. II. Intraobserver and interobserver reproducibility of measurements. Acta Ophthalmol Scand. 1998;76(5):546-549.

[21]. Dada T, Sihota R, Gadia R, Aggarwal A, Mandal S, Gupta V. Comparison of anterior segment optical coherence tomography and ultrasound biomicroscopy for assessment of the anterior segment. J Cataract Refract Surg. 2007;33(5):837-840.

[22]. Tello C, Liebmann J, Potash SD, Cohen H, Ritch R. Measurement of ultrasound biomicroscopy images: intraobserver and interobserver reliability. Invest Ophthalmol Vis Sci. 1994;35(9):3549-3552. 